\title{
Quimioterapia abreviada de 6 meses en tuberculosis pulmonar infantil \\ (Informe Preliminar)
}

Dr. Sergo lbánez Quevedo, Dr. Gustavo Ross Bravo Fundación Hospital Para Niños Josefina Martínez de Ferrari.

\begin{abstract}
A six months short-rsurse chemotherapy was performed on $\$ 4$ clijldren with proven pulmonan tuberculosis.

Accordingly un demonstration or nuw of tuberculosis bacili this malerial was divided into t wo proups both recieving twio months courses of daily therapy and four months of bix eekly ireatment. The first group, with posilive bacillus finding, recieved a reginen of SM - INH - RMP - PZ fur I wo months and SM- INH - PZ for four months; the second gmup wilh negative bacteriologiral findings, the same treatment except for SM which was not included.

TB. bacillus convertion was nbserved in all cases by the end of the firs I month of therapy and remained negutive in all patients except one child who reactivated by the fourth months due in primary resistance to SM and I.VH. study.

Important $X$-Rny improvement was observed in 37 cases, and presistance of sequelac in 9 at the end of the six months

No intolerance nor toxicity was observed in the children treated. and in 30 cases in which trangaminase sludies were undergone at the end of the first and second munthe of the dail trearment, they showed normal levels.

We shall continue the clinical, X-Ray and bacteriological follow-up of these patients and the results will be reported at a later date.

This study was possible due to the cooperation of different Hospitals of the Region Metropolitana who sent sheir patients to be intemed during the whole six mont bs period at the Hospital para Ninuos de la Fundaciún Josefina Martinez de Ferrai.
\end{abstract}

Numerosos estudios terapéuticos controlados han demostrado la eficacia de esquemas de Quimioterapia de 6 meses de duración en la tuberculosis pulmonar de adultos, basados en la eficacia de nuevas y poderogas drogas bactericidas. $1,2,3,4,5,6,7,0$

La aplicación de estas terapias abreviadas en los niños no han sido aún comunicadas en la literatura médica analizada.

En la Región Metropolitana se programó, de acuerdo a instrucciones del Ministerio de Salud, una experiencia piloto de tratamiento acortado en adultos y niños iniciada en enero de 1978 en los primeros y en junio del mismo año en los segundos.

Lo9 niños con diagnóstico de tuberculosis pulmonar efectuado en los distintos hospitales de esta Región, con tratamiento iniciado o sin él, fueron referidos al Hospital para Niños de la Fundación Josefina
Martínez de Ferrari para efectuar su quimioterapia, control clínico, bacterioḱgico y radiológico.

\section{MATERIAL Y METODO}

Conocido el becho que la gravedad de la tuberculosis pulmonar está en relación con la magnitud de la población bacilar que se evidencia por el hallazgo del Bacilo de Koch en la expectoración o contenido gástrico, se decidió tratar estos pacientes con dos esquemas diferentes, El primero, en el cual era posible demostrar el Bacilo de Koch en examen directo o cultivo y un segundo esquema en el cual esta verifcación no se cumplía o bjen el cultivo era informado como positivo con posterioridad a la íniciación del tratamiento. Tabla N. ${ }^{0} 1$. 
A. Etapa inicial, diaria: 2 meses.

SM 20-25 mg por kilo de peso

INH $10 \mathrm{mg}$ por kilo de peso

RMP 10 mg por kilo de peso

PZ $30 \mathrm{mg}$ por kilo de peso

B. Etapa intermitente, 2 veces por semana: 4 meses.

SM 20-25 mg por kilo de peso por día

INH $15 \mathrm{mg}$ por kilo de peso por día

PZ $\quad 50 \mathrm{mg}$ por kilo de peso por día

ESQUEMA II: Koch directo negativo

A. Etapa inicial, diaria: 2 meses.

INH $10 \mathrm{mg}$ por kilo de peso

RMP $10 \mathrm{mg}$ por kilo de peso

PZ 30 mg por kilo de peso

B. Etapa intermitente, 2 veces por sernana: 4 meses.

INH 15 mg por kilo de peso por día

\section{PZ 50 mg por kilo de peso por día}

Ingresaron a la experiencia 54 niños, 15 se trataron con el esquema I (Koch positivo) y $39 \mathrm{con}$ el esquema II (Koch negativo). De ellos, 8 aún están en tratamiento y 46 terminaron sus 6 meses de quimioterapia. (Tabla N. ${ }^{\circ} 2$.)

\section{TABLA N.*2}

\begin{tabular}{lccc} 
& $\begin{array}{c}\text { N. } \\
\text { Eocien- } \\
\text { tes }\end{array}$ & $\begin{array}{c}\text { En trata- } \\
\text { miento }\end{array}$ & $\begin{array}{c}\text { Tratam. } \\
\text { terminados }\end{array}$ \\
Esquema I & 15 & 3 & 12 \\
Total & 39 & 5 & 34 \\
\cline { 2 - 4 } & 54 & 8 & 46
\end{tabular}

Otros pacientes que efectuaron tratamiento ambulatorio en las distintas áreas de esta Región no han sido incluidos en el presente trabajo.

Referente a la extensión radiológica lesional se ha seguido la clasificación de la American Thoracic Society.

De los 54 casos estudiados 17 eran mínimos, 26 moderados y 11 avanzados. (Tabla N. ${ }^{\circ} 3$ ).

\begin{tabular}{|c|c|c|c|c|}
\hline & Mínima & $\begin{array}{l}\text { Mode- } \\
\text { rada }\end{array}$ & $\begin{array}{c}\text { Avan- } \\
\text { zada }\end{array}$ & $\begin{array}{c}\text { Total de } \\
\text { casas }\end{array}$ \\
\hline Esquema I & 0 & 4 & 11 & 15 \\
\hline Esquema II & 17 & 22 & 0 & 39 \\
\hline Total & 17 & 26 & 11 & 54 \\
\hline
\end{tabular}

Edades. La edad de los pacientes varió de dos meses a quince años. (Tabla N.*4.)

TABLA N. ${ }^{0} 4$

\begin{tabular}{cccc} 
Mínima & $\begin{array}{c}\text { Mode- } \\
\text { rada }\end{array}$ & $\begin{array}{c}\text { Avan- } \\
\text { zada }\end{array}$ & Total \\
5 & 6 & 3 & 14 \\
9 & 4 & 0 & 13 \\
3 & 16 & 8 & 27 \\
\hline 17 & 26 & 11 & 54
\end{tabular}

En el grupo de 0-2 años y en el de 6-15 años predominaron las lesiones de mayor extensión, en tanto que en el grupo de $2-6$ años las lesiones fueron menores.

En ambos grupos se efectuaron controles clinicos y observaciones de tolerancia y toxicidad a las drogas empleadas. Controles radiológicos al $4^{\circ}$ y $6^{\circ}$ mes. En los 15 niños tratados con Esquema I el estudio bacteriológico se efectuó mensualmente en forma de baciloscopía directa y cultivos de muestras de expectoración o contenido gástrico. En los 39 niños del Esquema II estos controles se efectuaron al término del $10^{\circ}$ y $6 .^{\circ}$ mes. El estudio de la tolerancia a drogas mostró que en el grupo analizado no se presentaron molestias gástricas, de hipersensibilidad ni toxicidad. (Tabla N. ${ }^{\circ} 5$.)

\section{TABLA N, ${ }^{\circ} 5$}

\section{CONTROLES DURANTE EL TRATAMIENTO}

ESQUEMA I: Clínico, tolerancia, toxicidad Bacteriológico mensual Radiológico 4. ${ }^{\circ}$ y $6 .^{\circ}$ mes

ESQUEMA II: Clínico, tolerancia, toxicidad Bacteriológico $1 .^{\circ}$ y $6 .^{\circ}$ mes Radiológico 4..$^{\circ}$ y $6{ }^{\circ}$ mes 
A todos los niños que han sezuido esta experiencia se han programado controles clínicos, radiológicos y bacteriológicos de acuerdo a la tabla N. ${ }^{0} 6$.

TABLA N. ${ }^{\circ} 6$

\section{CONTROL DE SEGUIMIENTO POST QUIMIOTERAPIA}

Clínico, Bacteriológico y Radiológico

Grupo I: $3 .^{\circ}-6 .^{\circ}-12 .^{\circ}-18 .^{\circ}$ mes post tratamiento

Grupo II: $3 .^{\circ}-6 .^{\circ}$ mes post quimioterapia

\section{RESULTADOS}

Control Bacteriológico. Todos los pacientes que presentaron baciloscopía positiva inicial negativizaron este resultado, ya, al primer mes y continuaron negativos excepto en un caso en que reaparece la baciloscopía y cultivo positivos al $4 .^{\circ}, 5 .^{\circ}$ y $6 .^{\circ} \mathrm{mes}$, lo que se consideró fracaso por resistencia primaria comprobada a Estreptomicina e Isoniacida. En los pacientes del esquema II con baciloscopía inicial negativa continuaron con igual resultado hasta el fin de su tratamiento. (Tabla N,${ }^{\circ}$ 7.)

\section{TABLA N $0^{\circ} 7$}

\section{CONTROL BACTERIOLOGICO DURANTE LA QUIMIOTERAPLA}

Todos negativizaron al primer mes.

Un caso del grupo I reaperecen baciloscopía y cultivo positivo al 4. ${ }^{\circ}$ mes (Fracaso por Resistencia Primaria a SM e INH).

Control Radiológico. Al término de la quimioterapia se observô regresión radiológica total en 34 casos, parcial en 3 y en 9 lesiones residuales importantes. (Tabla N. 8.)

\section{TABLA N,${ }^{\circ} \mathrm{g}$}

\section{CONTROL RADIOLOGICO AL TERMINO DE QUIMIOTERAPIA}

$\begin{array}{lr}\text { Regresión total } & 34 \\ \text { Regresión parcial } & 3 \\ \text { Lesión residual importante } & 9 \\ \text { Total } & 46\end{array}$

Tolerancia, Toxicidad e Hipersensibilidad. En el material estudiado no se observaron manifestaciones de intolerancia que modificaran el esquema terapéutico, sin embargo, en un caso que inició tratamiento acortado en un Hospital de la Región presentó toxicidad a Isoniacida y Rifampicina razón por la cual se le retiró del esquema y no fue trasladado a nuestro hospital. (Tabla N. ${ }^{a 9 .)}$

\section{TABLA N. ${ }^{\circ} 9$}

\section{TOLERANCIA A LAS DROGAS}

1. Estudio en 30 casos de Transaminasas al 0-1-2 meses. Todas normales.

2. Tolerancia gástrica: buena.

2 casos molestias superables.

3. Manifestaciones Hipersensibilidad: ninguna.

\section{RESUMEN}

1. Se analizan los resultados obtenidos con Quimioterapia acortada de 6 meses en una serie de 54 niños con tuberculosis pulmonar.

2. Según el hallazgo bacteriológico de bacilos tuberculosos se clasificó el material en dos grupos que recibjeron esquemas diferentes tanto en la etapa diaria de dos meses como en la intermitente de cuatro meses.

3. Los resultados son concluyentes en cuanto a la negativización del bacilo tuberculoso ya al mes de tratamiento y su no reaparición en los controles posteriores salvo en un caso de fracaso al $4 .^{\circ}$ mes por resistencia primaria.

4. Se evidenció importante regresión radiológica en 37 casos y persistencia de secuelas en 9 pacientes al término de los 6 meses de tratamiento.

5. No se observó intolerancia a los esquemas terapéuticos ni toxicidad a las drogas usadas, aun a la luz de estudios de Transaminasas en 30 casos analizados al $1 .^{\circ}$ y $2 .^{\circ}$ mes de tratamiento diario.

6. Se continuará los controles de estos pacientes de acuerdo a pautas determinadas y sus resultados serán comunicados posteriormente.

7. Este trabajo fue posible por la cooperación de los diferentes hospitales de la Región Metropolitana que enviaron sus pacientes a seguir la Quimioterapia internados en el Hospital para Niños de la Fundación Josefina Martínez de Ferrari durante todo el periodo del tratamiento. 


\section{REFERENCIAS}

1 COMISION DE TRATAMIENTO DE LA UNION INTERNACIONAL CONTRA LA TUBERCULOSIS. Orientaciones para la investigación sobre el tratamiento de lo tuberculosis. Baletín de lo L'niôn Internacional Contra la Tubereulosis, 53: 31 (1978).

${ }^{2}$ EAST AFRICAN/BRITISH MEDICAL RESEARCH COUNCILS. Controlled clinical trial of short-course (6-month) regimes of chemotherapy for treatment of tuberculosis. Lancet, 2: 237 (1974).

3 HONG KONG CHEST SERVICE/BRITSH MEDICAL RESEARCH COUNCIL. Controlled trial of 6-month and 8-month regimens in the treatment of pulmonary tubercukosis. American Review of Respiratory Disease, 118: 219 (1978).

$\rightarrow$ FOX W. \& MITCHISON D.A. State of the Art. Short-comerse chemotherapy for pulmonary tubercuhosis. American Review of Respiratory Disease. 111: 325 (1975).

${ }^{5}$ EAST AFRICAN/BRTTISH MEDICAL RESEARCH COUNCIL
STUDY. Restalts at 5 years of a controlled comparison of a 6-month ans a standard 18-month regimen of chemotherapy for pulmonary Intereulosis. American Review of Respiratory Disease, 116: 3 (1977).

${ }^{6}$ SECOND EAST AFRICAN/BRITISH MEDICAL RESEARCH COUNCIL STUDY. Conirolled clinical Irial of four 6-roooih regimes of chemotberapy for pulmonary tuberculosis. American Re. view of Respiratory Disease, 114: 471 (1976).

7 BRTTISH THORACIC AND TUBERCULOSIS ASSOCIATION. Short-course chemotherapy in pulmonary tuberculosis. Lance1, 11: 1102 (1976).

${ }^{9}$ HONG KONG TUBERCULOSIS TREATMENT SERVTCE BRITISH MEDICAL RESEARCH COUNCIL. Controlled Irial of 6-month and 9-rnonth regimes of daily and intermituent gtreptomycin plus isoniazid plus pyrazinamide for pulmonary tuberculosis in Hong Kang (The result up to 30 months). American Review of Respiratory Disease, 115: 727 (1975). 